\title{
Comparing the Catalytic Oxidation of Ethanol at the Solid-Gas and Solid-Liquid Interfaces over Size-Controlled Pt Nanoparticles: Striking Differences in Kinetics and Mechanism
}

\author{
Andras Sapi, ${ }^{\dagger}$ Fudong Liu $^{\dagger}$ Xiaojun Cai, $^{\dagger}$ Christopher M. Thompson, Hailiang Wang, Kwangjin An, \\ James M. Krier, and Gabor A. Somorjai* \\ Department of Chemistry, University of California, Berkeley, California 94720, United States \\ Materials Sciences and Chemical Sciences Divisions, Lawrence Berkeley National Laboratory, Berkeley, California 94720, United \\ States
}

\section{Supporting Information}

ABSTRACT: Pt nanoparticles with controlled size (2, 4, and $6 \mathrm{~nm}$ ) are synthesized and tested in ethanol oxidation by molecular oxygen at $60^{\circ} \mathrm{C}$ to acetaldehyde and carbon dioxide both in the gas and liquid phases. The turnover frequency of the reaction is $\sim 80$ times faster, and the activation energy is $\sim 5$ times higher at the gas-solid interface compared to the liquid-solid interface. The catalytic activity is highly dependent on the size of the Pt nanoparticles; however, the selectivity is not size sensitive. Acetaldehyde is the main product in both
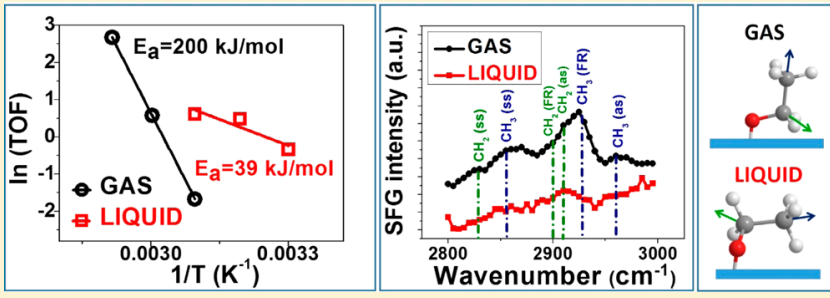
media, while twice as much carbon dioxide was observed in the gas phase compared to the liquid phase. Added water boosts the reaction in the liquid phase; however, it acts as an inhibitor in the gas phase. The more water vapor was added, the more carbon dioxide was formed in the gas phase, while the selectivity was not affected by the concentration of the water in the liquid phase. The differences in the reaction kinetics of the solid-gas and solid-liquid interfaces can be attributed to the molecular orientation deviation of the ethanol molecules on the Pt surface in the gas and liquid phases as evidenced by sum frequency generation vibrational spectroscopy.

KEYWORDS: Heterogeneous catalysis, ethanol oxidation, size control, platinum nanoparticles, sum frequency generation

$\mathrm{P}$ latinum nanoparticles with controlled size are promising candidates for heterogeneous catalytic processes. The activity and selectivity of catalytic reactions can be highly dependent on the size of the nanoparticles. ${ }^{1-3}$ For example, methanol oxidation by molecular oxygen over Pt nanoparticles with 1-6 nm sizes exhibited higher turnover frequency (TOF) values and higher selectivity toward carbon dioxide in the case of particles with larger sizes. ${ }^{4}$

Alcohol oxidation reactions are used in industrial processes for energy conversion and as starting materials for synthesis of organic chemicals as well as for pharmaceuticals. For example, several highly effective fuel cells are based on the complete oxidation of low molecular weight alcohols, ${ }^{5,6}$ while complex alcohols are used for production of drugs and fine chemicals. ${ }^{7}$

There are numerous studies focused on the catalytic oxidation of alcohols with molecular oxygen. Usually liquid phase reactions are performed at near-ambient temperatures, ${ }^{8}$ while elevated temperatures are used for gas phase alcohol oxidations. ${ }^{9}$ Tuning the activity and selectivity of the reactions by varying the experimental conditions such as pressure, temperature, and reactant concentration has been extensively studied. ${ }^{10,11}$ However, the effect of changing the phase from gas to liquid to the reaction performance under similar reaction conditions was only recently studied in our laboratory. We observed that the platinum catalyzed oxidation of isopropanol to acetone in gas and liquid phases showed dramatically different kinetics and mechanisms. ${ }^{12}$

In this letter, we investigate the catalytic oxidation of ethanol by molecular oxygen to acetaldehyde and carbon dioxide in both gas and liquid phases over Pt nanoparticles with controlled size in the range of $2-6 \mathrm{~nm}$. To compare the effect of the different medium phases, rate and activation energy of the reaction, as well as the $\mathrm{Pt}$ size effect and added water contribution were studied in both gas and liquid phases under similar reaction conditions. The turnover frequency is 2 orders of magnitude higher, and the activation energy is five times larger in the gas phase compared to the liquid phase. Larger platinum nanoparticles show higher catalytic activity compared to the smaller ones in both the gas and liquid phases. Acetaldehyde is the main product. Twice as much carbon dioxide is formed in the gas phase compared to the liquid phase. Added liquid water boosts the reaction in the liquid phase; however, water vapor acts as an inhibitor in the gas phase. The more water vapor was added, the more carbon

Received: September 15, 2014

Revised: October 15, 2014 

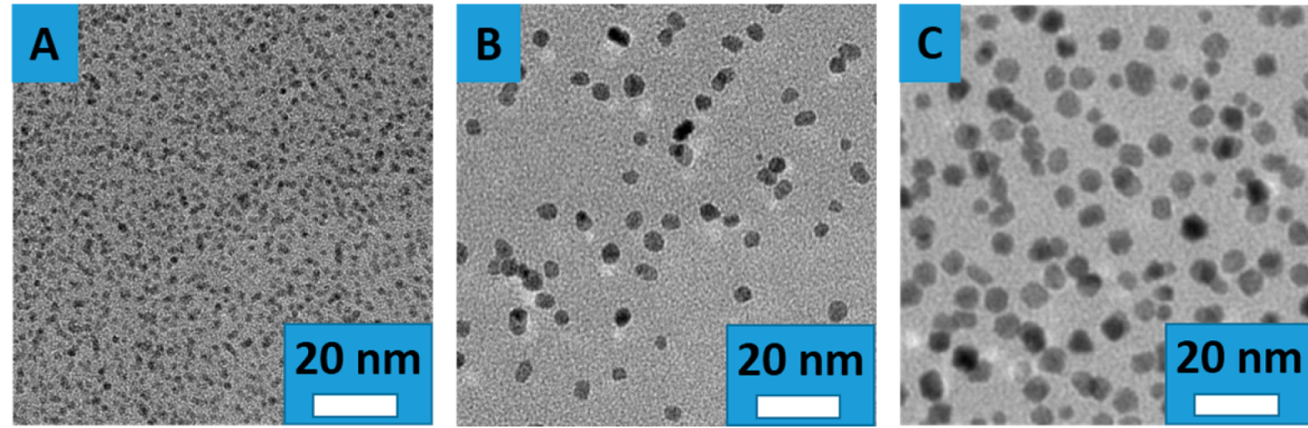

Figure 1. Typical TEM images of platinum nanoparticles with (A) 2, (B) 4, and (C) $6 \mathrm{~nm}$ average sizes.

dioxide was formed in the gas phase, while the selectivity did not change with the concentration of the water in the liquid phase. The differences in the reaction kinetics between the gas and liquid phases may be the results of the molecular orientation deviation of the ethanol molecules on the $\mathrm{Pt}$ surface in the gas and liquid phases as probed by sum frequency generation (SFG) vibrational spectroscopy.

Result and Discussion. Platinum nanoparticles with various sizes were synthesized by PVP-assisted polyol process in ethylene glycol $^{4,12,13}$ (see Supporting Information for details). The average sizes for the nanoparticles are $1.6 \pm 0.4$, $3.9 \pm 0.6$, and $5.5 \pm 0.9 \mathrm{~nm}$, respectively with a narrow size distributions (Figure S1, Supporting Information) as obtained from transmission electron microscopic (TEM) images (Figure 1). Pt nanoparticles with different sizes showed nearly spherical shapes.

Platinum nanoparticles were loaded into inert, MCF-17 mesoporous silica support for investigation of catalytic oxidation of ethanol by molecular oxygen in both gas and liquid phases. The gas phase reactions were performed in a gold-coated batch reactor, while a high pressure, Teflon-lined autoclave was used for liquid phase experiments (see Supporting Information for details). The products of the ethanol oxidation reactions were carbon dioxide and acetaldehyde as detected by gas chromatography.

For $6 \mathrm{~nm}$ Pt nanoparticles, the turnover frequency was 3.23 molecules $\cdot \mathrm{site}^{-1} \cdot \mathrm{s}^{-1}$ at $60{ }^{\circ} \mathrm{C}$ using 10 Torr of ethanol and 50 Torr of $\mathrm{O}_{2}$ in the gas phase (Figure $2 \mathrm{~A}$ ). When using pure liquid ethanol and pure $\mathrm{O}_{2}$ with the pressure of 1 atm at $60^{\circ} \mathrm{C}$, the turnover frequency was 2.06 molecules $\cdot s_{i t e}{ }^{-1} \cdot \mathrm{s}^{-1}$. The concentration of $\mathrm{O}_{2}$ is of the same order of magnitude ( $2.7 \mathrm{vs}$ $9.7 \mathrm{mM}$ ); however, the ethanol concentration was $\sim 3000$ times higher in the liquid phase compared to the gas phase due to the much higher density. In order to mitigate the contrast, the
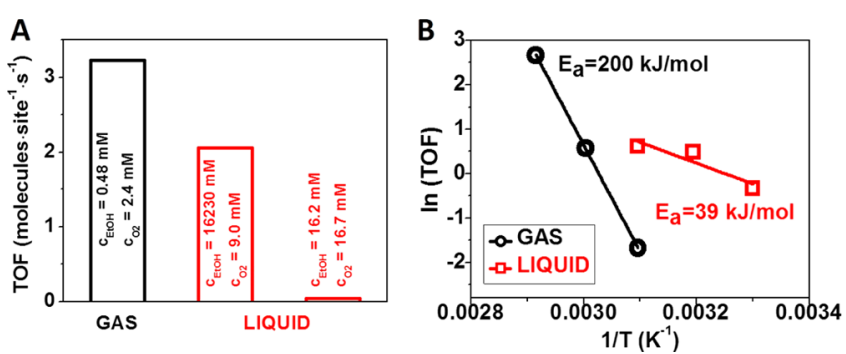

Figure 2. (A) TOF value comparison in the gas and liquid phases and (B) activation energy differences between the gas and liquid phases. Reaction is 80 times faster and the activation energy is 5 times higher in the gas phase compared to the liquid phase. ethanol was diluted with heptane to 1:1000 ratio in the liquid phase. Heptane is a neutral solvent as it showed a slight effect on reaction rate, size dependence, and activation energy in isopropanol oxidation under similar conditions. ${ }^{12}$ The turnover frequency in the liquid phase after the heptane dilution was 0.04 molecules $\cdot \operatorname{site}^{-1} \cdot \mathrm{s}^{-1}$, which exhibits $\sim 80$ times lower activity compared to the gas phase reaction under comparable concentration conditions. The results suggest almost 2 orders of magnitude slower kinetics at the liquid-solid interface than the gas-solid interface.

The activation energies in the gas and liquid phases also show differences as tested on $4 \mathrm{~nm}$ Pt nanoparticles. In the gas phase, the activation energy of the alcohol oxidation reaction was found to be $200 \mathrm{~kJ} / \mathrm{mol}$. However, in the liquid phase this value was $\sim 5$ times lower $(39 \mathrm{~kJ} / \mathrm{mol}$ ) (Figure $2 \mathrm{~B}$ ). The different activation energies suggest different reaction mechanisms in the liquid-solid and gas-solid interface.

In both the gas and liquid phases the catalytic activity was highly dependent on the size of the platinum nanoparticles in the $2-6 \mathrm{~nm}$ range. Higher turnover frequency was observed for larger nanoparticles (Figure $3 \mathrm{~A}$ ). In the gas phase at $60{ }^{\circ} \mathrm{C}$, the
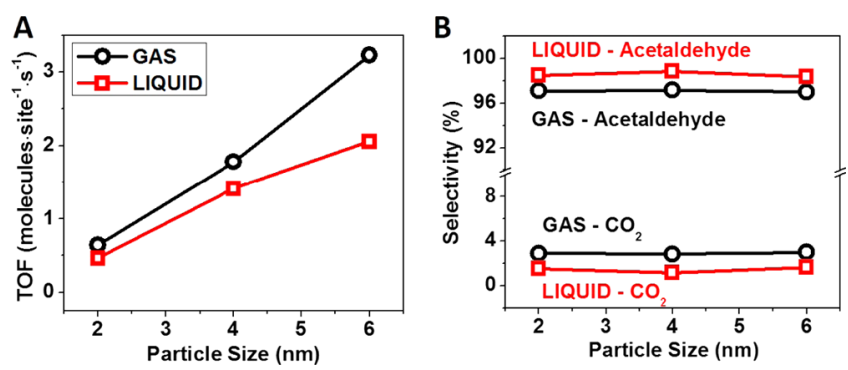

Figure 3. (A) Size-dependent turnover frequency and (B) corresponding selectivity results for both gas and liquid phases ethanol oxidation reactions over $\mathrm{Pt}$ nanoparticles. Larger $\mathrm{Pt}$ nanoparticles resulted in higher TOF for both gas and liquid phases. Acetaldehyde is the main product in both gas and liquid phases, though the carbon dioxide selectivity is 2 times higher in the gas phase compared to the liquid phase.

turnover frequency was 0.64 molecules $\cdot \mathrm{site}^{-1} \cdot \mathrm{s}^{-1}$ in the case of $2 \mathrm{~nm} \mathrm{Pt}$ nanoparticles, whose value is increased to 1.78 molecules $\cdot \mathrm{site}^{-1} \cdot \mathrm{s}^{-1}$ when $4 \mathrm{~nm} \mathrm{Pt}$ nanoparticles were used. The turnover frequency was as high as 3.23 molecules $\cdot \operatorname{site}^{-1} \cdot \mathrm{s}^{-1}$ for $6 \mathrm{~nm}$ Pt particles. In the range of $2-6 \mathrm{~nm}$, the TOF values increase by $\sim 5$ times for the gas phase reaction. With pure ethanol in the liquid phase at $60{ }^{\circ} \mathrm{C}$, the TOF was 0.46 molecules $\cdot \mathrm{site}^{-1} \cdot \mathrm{s}^{-1}$ for $2 \mathrm{~nm}$ Pt nanoparticles, 1.41 molecules. $\mathrm{site}^{-1} \cdot \mathrm{s}^{-1}$ for $4 \mathrm{~nm}$ Pt nanoparticles, and 2.06 molecules $\cdot \mathrm{site}^{-1}$. 
$\mathrm{s}^{-1}$ for $6 \mathrm{~nm}$ nanoparticles, respectively, showing an increase by $\sim 4.5$ times in the turnover frequencies similarly to the gas phase.

In both gas and liquid phases, the catalytic oxidation of ethanol with molecular oxygen yielded two products: carbon dioxide and acetaldehyde. Acetaldehyde was the dominant product in both phases, and the effect of Pt nanoparticle size on the product selectivity was insignificant (Figure 3B). In the gas phase, the selectivity toward carbon dioxide was $2.85-3 \%$, and the acetaldehyde selectivity was $97-97.15 \%$ in the $2-6 \mathrm{~nm}$ range. The liquid phase reactions show selectivities of 1.15$1.63 \%$ toward carbon dioxide and 98.37-98.85\% for acetaldehyde. The carbon dioxide selectivity is $\sim 2$ times higher in the gas phase compared to the liquid phase. However, both the gas-solid and liquid-solid interfaces show high selectivity toward acetaldehyde production.

In the case of the $4 \mathrm{~nm}$ Pt nanoparticles loaded on MCF-17 mesoporous silica support, the effect of water added to the reaction mixture was tested in both gas and liquid phases. When 1-10 Torr water vapor was introduced with 10 Torr of ethanol and 50 Torr of oxygen to the reaction chamber prior to gas phase alcohol oxidation, dramatic loss of catalytic activity was observed. One Torr of added water $($ ethanol/water $=10: 1)$ resulted in as low as $40 \%$ of the water-free reaction activity (Figure 4A). This value further decreased to $18 \%$ and $10 \%$
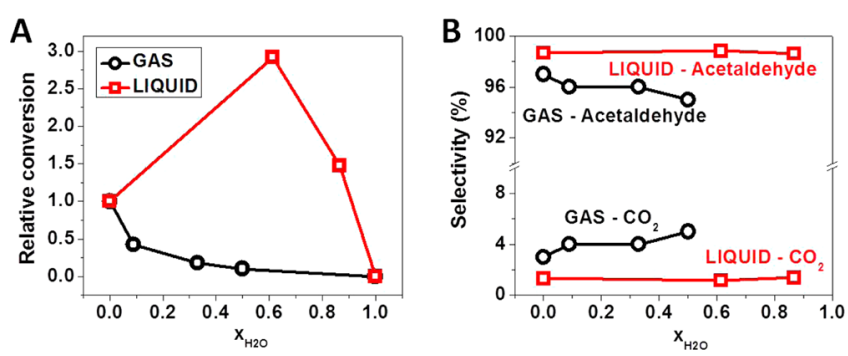

Figure 4. Effect of water addition on (A) conversion and (B) selectivity in both gas and liquid phases. Added water inhibits the reaction in the gas phase but boosts it in the liquid phase. The increase of the water concentration resulted in higher carbon dioxide selectivity in the gas phase, while the selectivity is insensitive to water concentration in the liquid phase.

when the ethanol to water ratios were $2: 1$ and $1: 1$, respectively. SFG studies showed that added water decreased the number of isopropanol molecules on the platinum surfaces, which shows that the water can block active sites for adsorption of alcohols (Figure S2, Supporting Information). However, in the liquid phase when $15 \mathrm{~mL}$ of ethanol/water mixture with a volume ratio of 2:1 was used, the catalytic activity was $\sim 3$ times higher compared to pure liquid ethanol. Further increment in the water concentration resulted in the decline of activity, however, at $1: 2$ ethanol/water volume ratio the activity was still $50 \%$ higher than in the water-free reaction. As the oxygen solubility in water is lower than in ethanol it is obvious that water has a promotion effect in the liquid phase. ${ }^{14}$ The markedly different effects of water on catalytic activity also suggests different reaction mechanisms in the gas-solid and liquid-solid interfaces.

In the gas phase, beside the catalytic activity, the selectivity was also influenced by the added water vapor. The water-free ethanol oxidation produced $3 \%$ of carbon dioxide and $97 \%$ of acetaldehyde (Figure 4B). At the ethanol to water ratio of 10:1, the carbon dioxide selectivity was increased to $4 \%$, which further reached $5 \%$ when the ethanol to water ratio was reduced to 1:1. As water competes for $\mathrm{Pt}$ active sites and is a product of the oxidation of ethanol, the increased carbon dioxide formation and the decreased acetaldehyde formation with the water addition in the gas phase is not expected. This phenomenon suggests that the ethanol oxidation toward carbon dioxide follow a different pathway other than the oxidation to acetaldehyde as an intermediate step. However, several studies of aqueous alcohol oxidation show that the oxidation occurs in two consecutive reactions; the oxidative dehydrogenation of alcohol followed by the oxidation of the aldehyde. ${ }^{8}$ In the liquid phase, the addition of water has no significant effect on the selectivity. The carbon dioxide selectivity is lower in the liquid phase compared to in the gas phase irrespective of the water concentration. The average selectivity is $1.3 \%$ and $98.7 \%$ toward carbon dioxide and acetaldehyde, respectively.

The effect of oxygen concentration on the catalytic activity and selectivity of the $4 \mathrm{~nm}$ Pt nanoparticles was also tested in both the gas and liquid phases. When 5-50 Torr of $\mathrm{O}_{2}$ gas was mixed with 10 Torr of ethanol in the gas phase and $1-5$ bar $\mathrm{O}_{2}$ was introduced to the pure liquid phase ethanol, the rate of oxidation of ethanol increased almost linearly with increasing $\mathrm{O}_{2}$ gas pressure in both the gas and liquid phases. The reaction order for oxygen was 1.23 and 0.74 in the gas and liquid phases, respectively (Figure S3A, Supporting Information). These values indicate first-order reaction kinetics with respect to molecular $\mathrm{O}_{2}$ in both the gas and liquid phases. Similar kinetics were observed in the liquid-phase oxidation of ethanol ${ }^{15}$ and the electrooxidation of 2-propanol. ${ }^{16}$

Changing the pressure of oxygen in the gas phase ethanol oxidation reaction also has effects on the selectivity. When the oxygen pressure was reduced from 50 Torr to 20 and 5 Torr, the selectivity toward carbon dioxide increased from $2.9 \%$ to $3 \%$ and $10 \%$ (Figure S3B, Supporting Information), respectively, which has not been expected as the increase of oxygen concentration usually leads to increased selectivity toward complete oxidation. This phenomenon supports the possibility of a different pathway of ethanol oxidation toward carbon dioxide as suggested in the studies of water effect described above. The effect of change of the oxygen concentration in the liquid phase was negligible. The selectivity toward carbon dioxide was in the range of $1.32-1.86 \%$ when the pure liquid phase ethanol was pressurized with 1-5 bar of oxygen.

SFG vibrational spectroscopy was used on electron beam deposited Pt films to explore the surface species of ethanol oxidation under reaction conditions. The SFG studies were carried out in a flow reactor (see Supporting Information for more details). Figure 5A shows the SFG spectra of ethanol oxidation on electron beam deposited $\mathrm{Pt}$ film at $60{ }^{\circ} \mathrm{C}$ in both gas and liquid phases. Three characteristic features of the $\mathrm{CH}$ modes in $\mathrm{CH}_{3}$ functional group are clearly present in the gas phase spectrum, including symmetric stretch at $\sim 2860 \mathrm{~cm}^{-1}$, Fermi resonance at $\sim 2930 \mathrm{~cm}^{-1}$, and asymmetric stretch at $\sim 2960 \mathrm{~cm}^{-1}$. The peak at $\sim 2830 \mathrm{~cm}^{-1}$ is the symmetric $\mathrm{CH}$ stretch for $\mathrm{CH}_{2}$ functional group. Two weak shoulders at $\sim 2900$ and $\sim 2911 \mathrm{~cm}^{-1}$ are the Fermi resonance and asymmetric $\mathrm{CH}$ modes for $\mathrm{CH}_{2}$, respectively.

The liquid phase reaction showed different vibrational features in the SFG spectrum. First, the vibrational modes in the $\mathrm{CH}$ region are much weaker in the liquid phase than in the gas phase under reaction conditions due to the differences in refraction indices of the gas and liquid phase ethanol. The strongest peak in $\mathrm{CH}$ region in the liquid phase spectrum is the 


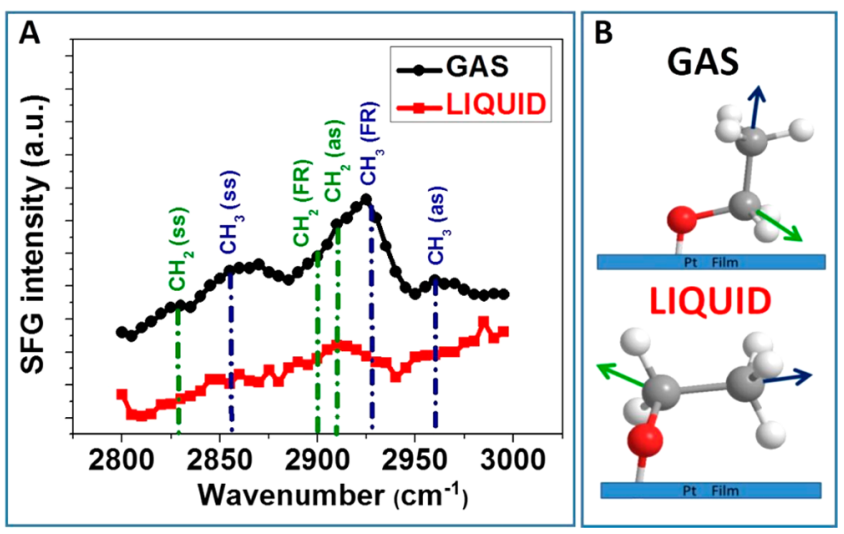

Figure 5. (A) SFG vibrational spectroscopy of ethanol oxidation reaction on electron beam deposited $\mathrm{Pt}$ film at $60{ }^{\circ} \mathrm{C}$ in both gas and liquid phases. (B) Schematic diagrams of differences in ethanol adsorption on the Pt film in gas and liquid phases under reaction conditions.

$\mathrm{CH}_{2}$ asymmetric peak at $\sim 2911 \mathrm{~cm}^{-1}$. Considering the $p p p$ polarization combination and the selection rule of the SFG spectrum, they strongly suggests that the $\mathrm{C}-\mathrm{CH}_{3}$ bond in ethanol stays more perpendicular to the $\mathrm{Pt}$ surface in the gas phase; however, the same bond is parallel to the surface in the liquid phase (Figure 5B). The different orientation of the adsorbed ethanol molecule on the $\mathrm{Pt}$ film under reaction conditions at the gas-solid and the liquid-solid interfaces can be responsible for the striking differences in kinetics and mechanism as found in the case of the turnover frequency, selectivity, activation energy, and effect of water on ethanol oxidation on Pt nanoparticles with different sizes.

Huge differences in kinetics and mechanisms were found in isopropanol oxidation reaction under similar condition using $\mathrm{Pt}$ nanoparticles with controlled sizes. ${ }^{12}$ The SFG studies and computational calculations suggested different orientation of the methyl groups connected to the $\alpha$-carbon atom. However, in the case of isopropanol the methyl groups are more likely parallel to the surface in the gas phase and at an angle away from the metal surface in the liquid phase. The differences in the number of carbon atoms and the localization of the $\mathrm{C}-\mathrm{OH}$ bond and the even-odd rule known from $\mathrm{SFG}^{17,18}$ can contribute to the different way of orientation of the isopropanol and ethanol molecules on the Pt surface.

Conclusions. Pt nanoparticles with controlled size (2, 4, and $6 \mathrm{~nm}$ ) were tested in ethanol oxidation with molecular oxygen at $60{ }^{\circ} \mathrm{C}$ both in the gas and liquid phases. The turnover frequency was $\sim 80$ times higher, and the activation energy was $\sim 5$ times higher at the gas-solid interface compared to the liquid-solid interface. The catalytic activity is highly dependent on, but the selectivity is not sensitive to, the size of the $\mathrm{Pt}$ nanoparticles. Acetaldehyde is the main product in both media, while twice as much carbon dioxide was observed in the gas phase compared to the liquid phase. Added water boosts the reaction in the liquid phase and acts as an inhibitor in the gas phase. The more water vapor was added, the more carbon dioxide was formed in the gas phase, while the selectivity was not dependent on water concentration in the liquid phase. The differences in the reaction kinetics at the gas-solid and liquidsolid interfaces can be attributed to the change of the molecular orientation of the ethanol molecules on the Pt surface in the gas and liquid phases. This work shows that tuning of catalytic activity and selectivity is possible by tuning the size of the $\mathrm{Pt}$ nanoparticles and by changing the phase of the reactants.

\section{ASSOCIATED CONTENT}

\section{S Supporting Information}

Experimental details of Pt nanoparticle synthesis, SFG studies, catalyst preparation, catalytic tests, size distribution of $\mathrm{Pt}$ nanoparticles, and SFG for added water and oxygen order tests. This material is available free of charge via the Internet at http://pubs.acs.org.

\section{AUTHOR INFORMATION}

\section{Corresponding Author}

*E-mail: somorjai@berkeley.edu.

\section{Author Contributions}

${ }^{\dagger}$ These authors contributed equally to this work.

Notes

The authors declare no competing financial interest.

\section{ACKNOWLEDGMENTS}

This work was supported by the U.S. Department of Energy, Office of Science, Division of Chemical Sciences, Geological and Biosciences under contract No. DE-AC02-05CH11231.

\section{REFERENCES}

(1) Rioux, R.; Hsu, B.; Grass, M.; Song, H.; Somorjai, G. A. Catal. Lett. 2008, 126, 10.

(2) Pushkarev, V. V.; Musselwhite, N.; An, K. J.; Alayoglu, S.; Somorjai, G. A. Nano Lett. 2012, 12, 5196.

(3) Kuhn, J. N.; Huang, W.; Tsung, C.-K.; Zhang, Y.; Somorjai, G. A. J. Am. Chem. Soc. 2008, 130, 14026.

(4) Wang, H.; Wang, Y.; Zhu, Z.; Sapi, A.; An, K.; Kennedy, G.; Michalak, W. D.; Somorjai, G. A. Nano Lett. 2013, 13, 2976.

(5) Gauthier, E.; Benzinger, J. B. Electrochim. Acta 2014, 128, 238.

(6) Reed, T. B.; Lerner, R. M. Science 1973, 28, 1299.

(7) Coleman, M. G.; Brown, A. M.; Bolton, B. A.; Guan, H. R. Adv. Synth. Catal. 2010, 352, 967.

(8) Jelemensky, L.; Kuster, B. F. M.; Marin, G. B. Catal. Lett. 1995, $30,269$.

(9) Gallezot, P. Catal. Today 1997, 37, 405.

(10) Tojo, G.; Fernández, M. Oxidation of Alcohols to Aldehydes and Ketones; Springer: New York, 2006.

(11) Kervinen, K.; Lahtinen, P.; Repo, T.; Svahn, M.; Leskela, M. Catal. Today 2002, 75, 183.

(12) Wang, H.; Sapi, A.; Thompson, C. M.; Liu, F.; Zherebetskyy, D.; Krier, J. M.; Carl, L. M.; Cai, X.; Wang, L.-W.; Somorjai, G. A. J. Am. Chem. Soc. 2014, 136, 10515.

(13) Alayoglu, S.; Aliaga, C.; Sprung, C.; Somorjai, G. A. Catal. Lett. 2011, 141, 914.

(14) Battino, R.; Rettich, T. R.; Tominaga, T. J. Phys. Chem. Ref. Data 1983, 12, 163.

(15) Hsu, V. S.-H.; Ruether, J. A. Ind. Eng. Chem. Process Des. Dev. 1978, 17, 524.

(16) DiCosimo, R; Whitesides, G. M. J. Phys. Chem. 1989, 93, 768.

(17) Whitesides, G. M.; Laibinis, P. E. Langmuir 1990, 6, 87.

(18) Tao, F.; Bernasek, S. L. Chem. Rev. 2007, 107, 1408. 